\title{
Mesenteriale Ischämie und vaskuläre Störungen
}

\author{
Ernst Klar ${ }^{a}$ Hans-Henning Eckstein ${ }^{b} \quad$ Karlheinz Hauenstein ${ }^{c}$ \\ a Abteilung für Allgemeine, Thorax-, Gefäß- und Transplantationschirurgie, Chirurgische Klinik und Poliklinik, Universität Rostock, \\ ${ }^{b}$ Abteilung für Gefäßchirurgie, Chirurgische Klinik, Klinikum rechts der Isar, Technische Universität München, \\ c Zentrum für Radiologie, Universität Rostock, Deutschland
}

Mit dem vorliegenden Themenheft verfolgen die Herausgeber ein besonderes Anliegen. Die Mortalität der akuten mesenterialen Ischämie persistiert seit Jahrzehnten auf hohem Niveau. Der Grund hierfür liegt in einer Verschleppung der Diagnose. Das Krankheitsbild an sich ist selten, das typische symptomfreie Intervall erschwert die Erkennung der lebensbedrohenden Situation. Der Patient wäre gerade in dieser Zeitphase der Erkrankung durch sofortige Restitution der mesenterialen Durchblutung zu retten. Durch die Schmerzarmut und den scheinbar stabilen Zustand baut sich bei der primären Evaluation des «unklaren Abdomens» eine Barriere gegenüber invasiver Diagnostik oder gar einer chirurgischen Exploration auf. Es besteht die Gefahr, sich in unspezifischer Umfelddiagnostik und Beobachtung zu verlieren, wodurch das Zeitfenster zum Darmerhalt verlassen wird.

Quo ad vitam ist die Erfassung der Erkrankung vor Auftreten einer Peritonitis entscheidend. In diesem Stadium erfordert die Verdachtsdiagnose «mesenteriale Ischämie» große klinische Erfahrung und basiert auf einem charakteristischen Risikoprofil der betroffenen Patienten; sie zwingt zu unmittelbaren Konsequenzen. Die höchste Sensitivität besitzt die Angiographie. Weitere Vorteile der Untersuchung sind die präzise OP-Planung sowie die Möglichkeit, nach Embolektomie häufig auftretende Spasmen durch selektive Vasodilatation über den liegenden Katheter zu durchbrechen. Die Reperfusion grenzwertig durchbluteter Darmabschnitte kann hierdurch verbessert werden. Dies gilt besonders für die nichtokklusive Ischämie. Kann aufgrund der Infrastruktur eine Angiographie nicht innerhalb $1 \mathrm{~h}$ durchgeführt werden, muss eine explorative Laparotomie zum sicheren Ausschluss der Ischämie bzw. zur rechtzeitigen Therapie erfolgen. Dieses strikte Schema ist darauf ausgerichtet, möglichst alle betroffenen Patienten schnell und sicher zu erfassen, besitzt aber den Nachteil einer potentiell hohen Zahl negativer Befunde. Ohne Zweifel muss die Computertomographie aufgrund der rasanten Qualitätssteigerung der letzten Jahre in diesem Kontext neu bewertet werden. Eine Beurteilung der zentra- len Abschnitte der Arteria mesenterica superior ist mittels Mehrzeilen-Spiral-CT zuverlässig möglich, so dass eine zentrale Embolie sicher nachgewiesen oder ausgeschlossen werden kann.

Die mesenteriale Ischämie auf dem Boden einer venösen Thrombose ist gegenüber der akuten arteriellen Perfusionsstörung oft maskiert und unspezifisch. Tritt eine Dünndarmnekrose auf, so ist diese meist segmental und durch eine umschriebene Resektion ohne Gefährdung des Patienten therapierbar. Die klare Kenntnis von den unausweichlichen Spätfolgen der Portal- und Mesenterialvenenthrombose im Sinne der portalen Hypertension verpflichtet heute jedoch zur akuten, interventionellen Rekanalisierung des Pfortadersystems. Diese erfolgt in den allermeisten Fällen über einen TIPS-Zugang transjugulär mit Fragmentation und lokaler Lyse des Thrombus. Muss ein Dünndarmsegment reseziert werden, so kann eine antegrade Lyse über einen Katheter, den der Chirurg einbringt, aufaddiert werden. Entscheidend ist die hohe Expertise eines interventionellen Radiologen. Diese Technik wird an nur wenigen Zentren beherrscht, an die die betroffenen Patienten unbedingt sekundär überwiesen werden sollten. Die venöse Thrombektomie durch den Gefäßchirurgen ist aufgrund der Intimaläsion und der hohen Rate von Rethrombosen eine Ausnahmeindikation.

In dem vorliegenden Themenheft stellen herausragende Experten des jeweiligen Fachgebiets die zentralen Aspekte der mesenterialen Ischämie umfassend dar. Die Beiträge machen deutlich, dass eine Abklärung ohne Verzögerung und eine fachübergreifende Infrastruktur zwischen Gastroenterologen, interventionellen Radiologen sowie Gefäß- und Viszeralchirurgen Voraussetzungen dafür sind, die hohe Mortalität der mesenterialen Ischämie zu senken und bei venöser Manifestation die Patienten vor den deletären Folgen der portalen Hypertension zu bewahren. Das interdisziplinäre Gespräch stellt besonders eindrücklich dar, wie die differenzierten Überlegungen der Einzeldisziplinen zu einem Gesamtkonzept dieses komplexen Krankheitsbilds verschmelzen.

\begin{tabular}{ll}
\hline KARGER & ( ) 2006 S. Karger GmbH, Freiburg \\
Fax +49 7614520714 & Accessible online at: \\
$\begin{array}{l}\text { E-mail Information@Karger.de } \\
\text { www.karger.com }\end{array}$ & www.karger.com/cga
\end{tabular}

Prof. Dr. med. Ernst Klar

Abteilung für Allgemeine, Thorax-, Gefäß- und Transplantationschirurgie

Chirurgische Klinik und Poliklinik, Universität Rostock

Schillingallee 35, 18057 Rostock, Deutschland

Tel. +49 381494 60-01, Fax -02

E-mail ernst.klar@med.uni-rostock.de 